\title{
Модель терагерцового квантово-каскадного лазера на основе двумерного плазмона
}

\author{
(C) А.А. Дубинов ${ }^{1,2}$, В.Я. Алешкин ${ }^{1,2}$ \\ ${ }^{1}$ Институт фризики микроструктур Российской академии наук, \\ 603950 Нижний Новгород, Россия \\ ${ }^{2}$ Нижегородский государственный университет им. Н.И. Лобачевского, \\ 603950 Нижний Новгород, Россия \\ E-mail: sanya@ipmras.ru \\ Поступила в Редакцию 12 апреля 2021 г. \\ В окончательной редакции 19 апреля 2021 г. \\ Принята к публикации 19 апреля 2021 г.
}

\begin{abstract}
Проведен расчет характеристик двумерного плазмона, усиливаемого активной средой на основе терагерцовой квантово-каскадной структуры. Показано, что для реалистичных параметров структур GaAs/AlGaAs с квантовыми ямами (подвижность $2 \cdot 10^{5} \mathrm{~cm}^{2} \cdot \mathrm{B}^{-1} \cdot \mathrm{c}^{-1}$ при концентрации электронов $5 \cdot 10^{11} \mathrm{~cm}^{-2}$ и при температуре до $77 \mathrm{~K}$ ) коэффициент усиления двумерного плазмона может достигать $1500 \mathrm{~cm}^{-1}$ на частоте 2.3 ТГц. Из-за сильной локализации электрического поля плазмона около квантовой ямы для усиления необходимо лишь несколько каскадов активной среды.
\end{abstract}

Ключевые слова: плазмон, квантово-каскадный лазер, терагерцовый диапазон частот, квантовая яма.

DOI: $10.21883 /$ FTP.2021.10.51435.34

\section{1. Введение}

К настоящему времени в терагерцовом (ТГц) диапазоне частот $(1-5$ ТГц) лидирующее место среди компактных источников стимулированного излучения занимают монополярные квантовые каскадные лазеры (ККЛ) [1]. Однако они представляют собой полупроводниковые гетероструктуры, содержащие сотни каскадов, каждый из которых состоит из нескольких квантовых ям (КЯ) и барьеров разной толщины и состава. Общая толщина активной области в ТГц ККЛ при использовании двойного металлического волновода, либо волновода на основе поверхностного плазмона, составляет обычно $\sim 10$ мкм (порядка длины волны излучения в среде) [1]. Минимальная толщина активной области ККЛ, излучающего на частоте 3.4 ТГц до температуры $80 \mathrm{~K}$, составила 1.75 мкм [2]. Поэтому чрезвычайно сложная зонная схема каскадных структур и высокие требования по контролю параметров на большой ширине активной области сдерживают распространение и использование ТГц ККЛ.

В данной работе рассмотрена модель ТГц ККЛ на основе двумерного (2D) плазмона, локализованного около КЯ с большой концентрацией электронов. Как известно, 2D-плазмон может быть локализован в области, много меньшей длины волны плазмона в вакууме [3]. Следовательно, можно подобрать параметры КЯ (состав, толщину и легирование) для того, чтобы необходимая активная область ТГц ККЛ на основе 2D-плазмона была много меньше длины волны излучения в среде. Это должно привести к тому, что вместо сотен каскадов для работы такого ТГц ККЛ необходимы будут только десятки, а то и всего несколько каскадов, что сильно упростит создание такого лазера. В данной работе проведены оценки для параметров гетероструктуры такого ТГц
ККЛ на основе 2D-плазмона и показана реалистичность его создания.

\section{2. Модель расчета}

Рассмотрим следующую структуру (рис. 1): КЯ $\mathrm{GaAs} / \mathrm{AlGaAs}$ c большой концентрацией электронов расположена между двумя слоями активной среды ТГц ККЛ. Вдоль этой КЯ может распространяться 2D-плазмон [4], распределение реальной части $z$-компоненты электрического поля в котором также по-

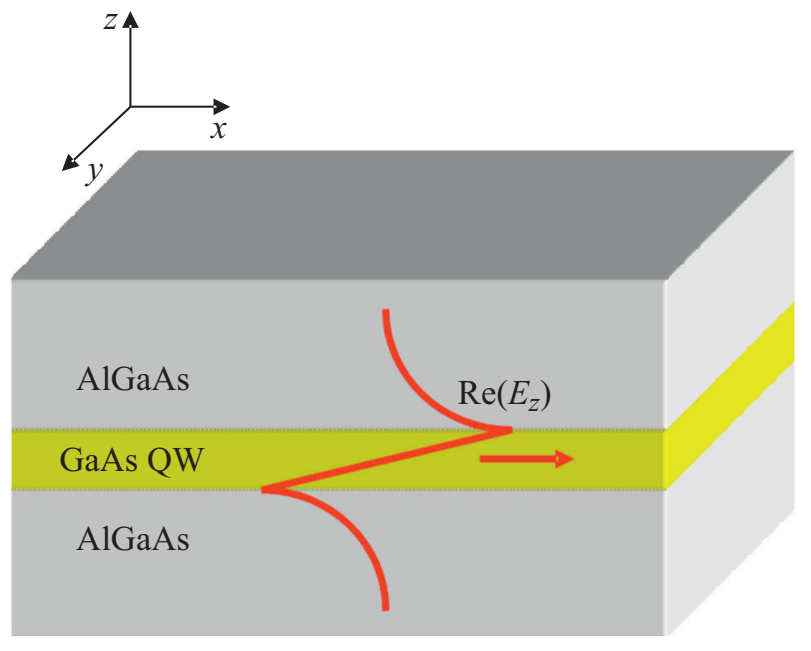

Рис. 1. Схема GaAs/AlGaAs-структуры с 2D-плазмоном. Красной кривой показана реальная часть $z$-компоненты электрического поля в 2D-плазмоне. (Цветной вариант рисунка представлен в электронной версии статьи). 
казано на рис. 1. Только эту компоненту электрического поля может усиливать активная среда ТГц ККЛ.

Запишем закон дисперсии 2D-плазмонов. В этом случае для бесконечно тонкой КЯ (это приближение хорошо выполняется, так как толщина КЯ обычно много меньше, чем длина волны ТГц излучения) уравнение для определения спектра приобретает вид (формула Стерна [3]):

$$
1+(2 \pi / \kappa) \chi(q, \omega) \sqrt{q^{2}+\kappa(\omega / c)^{2}}=0
$$

где $\kappa$ - диэлектрическая проницаемость, $\omega$ и $q-$ частота и волновой вектор плазмона, $c$ - скорость света в вакууме, $\chi(q, \omega)$ - восприимчивость.

Вычислим восприимчивость в простейшем случае, когда заполнена одна зона, двукратно вырожденная по спину, и закон дисперсии электронов квадратичен (с эффективной массой $m$ ). Выражение для восприимчивости можно записать в виде

$$
\chi(q, \omega)=\left(e^{2} / 2 \pi q^{2}\right)[F 1+F 2+F 3+F 4],
$$

где

$$
\begin{gathered}
F 1=\left(\left|k_{1}\right| / k_{1}\right) \int_{0}^{A 1} f(k) d k^{2} / \sqrt{k_{1}^{2}-Q^{2}}, \\
F 2=i \int_{A 1}^{\infty} f(k) d k^{2} / \sqrt{Q^{2}-k_{1}^{2}}, \\
F 3=\int_{0}^{A 2} f(k) d k^{2} / \sqrt{k_{2}^{2}-Q^{2}}, \\
F 4=-i \int_{A 2}^{\infty} f(k) d k^{2} / \sqrt{Q^{2}-k_{2}^{2}},
\end{gathered}
$$

и где $Q=\hbar^{2} q k / m, \hbar-$ постоянная Планка, $e-$ заряд электрона, $k_{1}=\hbar^{2} q^{2} / 2 m-\hbar(\omega+i v)$, $k_{2}=\hbar^{2} q^{2} / 2 m+\hbar(\omega+i v), \quad A 1=m\left|k_{1}\right| /\left(\hbar^{2} q\right), \quad A 2=$ $=m\left|k_{2}\right| /\left(\hbar^{2} q\right), f(k)$ - функция распределения ФермиДирака, $v$ - частота релаксации импульса. В простейшем приближении $v=e /(m \mu)$, где $\mu-$ подвижность электронов.

Учет усиления в активной области, окружающей КЯ с большой концентрацией электронов $N$, может быть проведен через добавок отрицательной мнимой части к диэлектрической проницаемости среды:

$$
\kappa=\kappa_{0}-i c \sqrt{\kappa_{0}} G / \omega,
$$

где $\kappa_{0}-$ статическая диэлектрическая проницаемость среды, $G$ - коэффициент усиления в активной области. Частотную зависимость $G$ можно аппроксимировать лоренцевым контуром:

$$
G=G_{\max } /\left\lfloor 1+(\omega-\Omega)^{2} / \Gamma^{2}\right\rfloor,
$$

где $G_{\max }$ - максимальный коэффициент усиления, $\Omega$ резонансная частота усиливаемого оптического перехода активной области, Г - параметр уширения спектра усиления активной области.

\section{3. Обсуждение и результаты расчетов}

Для примера рассмотрим активную область ТГц ККЛ на основе гетероструктуры GaAs/AlGaAs. Для расчетов будем использовать параметры ККЛ, генерирующего на частоте $\Omega / 2 \pi \sim 2.3$ ТГц $(\hbar \Omega \sim 10 \mathrm{мэВ)}$ [5]. В этом случае $\kappa_{0}=12.9, m=0.067$ массы свободного электрона, $G_{\max } \sim 50 \mathrm{~cm}^{-1}$ для температуры $T=77 \mathrm{~K}$, $\hbar \Gamma \sim 5$ мэВ [5]. Ранее экспериментально было показано, что в структурах $\mathrm{GaAs} / \mathrm{AlGaAs}$ с КЯ при температуре до $77 \mathrm{~K} \mu$ может достигать значений в $2 \cdot 10^{5} \mathrm{~cm}^{2} \cdot \mathrm{B}^{-1} \cdot \mathrm{c}^{-1}$ при $N$ до $5 \cdot 10^{11} \mathrm{~cm}^{-2}[6]$.

Используя формулы (1)-(8), были вычислены частотные зависимости эффективного показателя распространения $(S=c \operatorname{Re}(q) / \omega)$ и коэффициента поглощения $(\alpha=2 \operatorname{Im}(q))$ 2D-плазмона для нескольких значений $\mu$ и $N$.

Результаты расчетов приведены на рис. 2 и 3. Из рис. 2 видно, что при фиксированном значении $N$ увеличение $\mu$, а следовательно, уменьшение $v$ приводит к появлению области частот, где $\alpha$ 2D-плазмона становится отрицательным (появляется усиление), причем

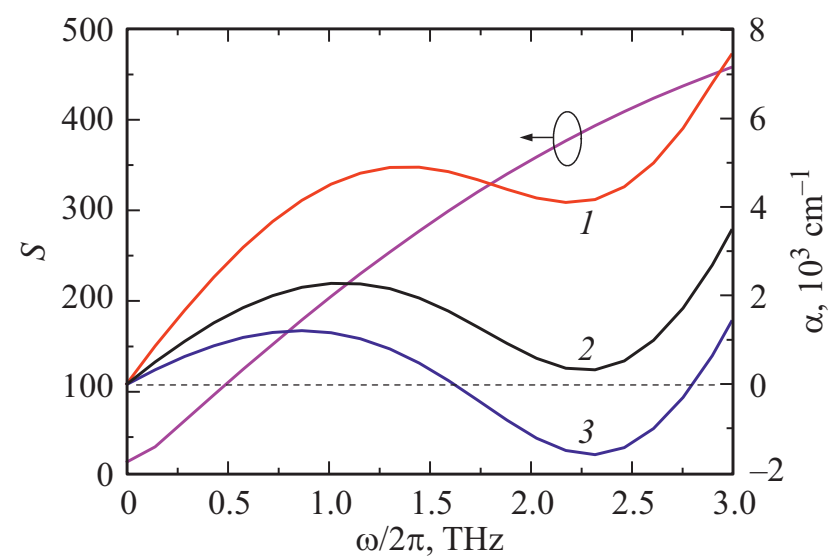

Рис. 2. Частотные зависимости $S$ и $\alpha$ 2D-плазмона для трех значений $\mu\left(\mathrm{cm}^{2} \cdot \mathrm{B}^{-1} \cdot \mathrm{c}^{-1}\right): 1-10^{5}, 2-1.5 \cdot 10^{5}, 3-2 \cdot 10^{5}$. $N=5 \cdot 10^{11} \mathrm{~cm}^{-2}, T=77 \mathrm{~K}$

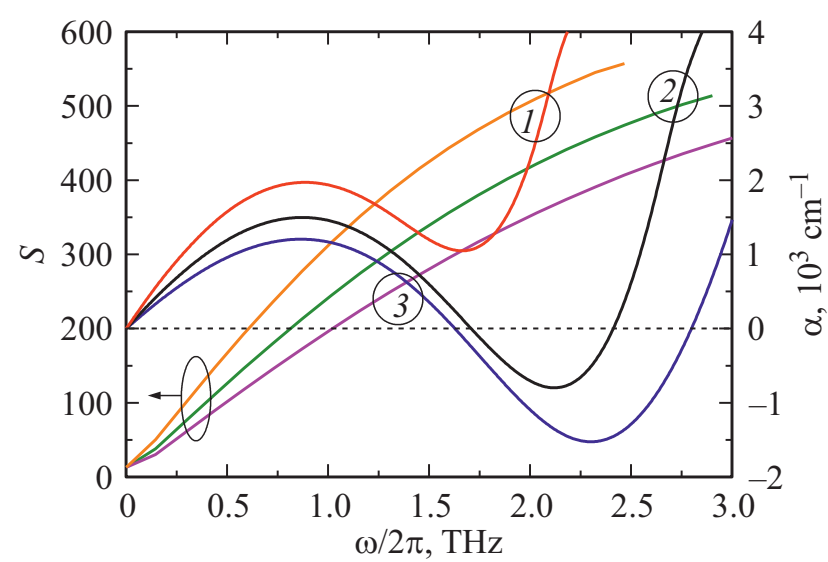

Рис. 3. Частотные зависимости $S$ и $\alpha$ 2D-плазмона для трех значений $N\left(\mathrm{~cm}^{-2}\right): 1-3 \cdot 10^{11}, 2-4 \cdot 10^{11}, 3-5 \cdot 10^{11}$. $\mu=2 \cdot 10^{5} \mathrm{~cm}^{2} \cdot \mathrm{B}^{-1} \cdot \mathrm{c}^{-1}, T=77 \mathrm{~K}$. 
коэффициент усиления достигает значения $1500 \mathrm{~cm}^{-1}$, что недостижимо для ТГц ККЛ с традиционными волноводами. При этом $S$ практически не зависит от $\mu$. Из рис. 3 видно, что при фиксированной $\mu$ существует критическая концентрация электронов в КЯ, при превышении которой появляется область усиления. Также можно видеть, что $S$ существенно зависит от $N$ : при увеличении $N$ он уменьшается. Отметим, что $S$ достигает огромных значений. Например, для $N=5 \cdot 10^{11} \mathrm{~cm}^{-2}$ на $\omega / 2 \pi=2.3$ ТГц он равен 400 , что говорит о сильной локализации электрического поля плазмона около КЯ (размер локализации в этом случае $\sim 600$ нм). Для ККЛ из работы [5] размер одного каскада $\sim 57$ нм, а следовательно, в структуре ККЛ лишь с десятью такими каскадами можно было бы добиться значительного усиления и генерации ТГц излучения.

\section{4. Заключение}

В работе рассмотрена модель терагерцового квантово-каскадного лазера на основе двумерного плазмона. Показано, что для лазерной структуры с $\mathrm{GaAs} / \mathrm{AlGaAs}$ квантовой ямой, обладающей подвижностью $2 \cdot 10^{5} \mathrm{~cm}^{2} \cdot \mathrm{B}^{-1} \cdot \mathrm{c}^{-1}$ при концентрации электронов $5 \cdot 10^{11} \mathrm{~cm}^{-2}$ и температуре до $77 \mathrm{~K}$, коэффициент усиления двумерного плазмона может достигать $1500 \mathrm{~cm}^{-1}$ на частоте 2.3 ТГц. При этом размер локализации электрического поля плазмона около квантовой ямы составляет $\sim 600$ нм, что много меньше реализованного ранее [2]. Отметим также, что из-за огромных величин эффективного показателя распространения и усиления длина ТГц ККЛ может быть тоже небольшой ( 100 мкм). Это позволит сделать такой лазер сверхкомпактным.

\section{Финансирование работы}

Работа выполнена при поддержке Фонда развития теоретической физики и математики,Базис“ (№ 19-1-2-12-1) и Министерства науки и высшего образования Российской Федерации (№ 0030-2021-0019).

\section{Конфликт интересов}

Авторы заявляют, что у них нет конфликта интересов.

\section{Список литературы}

[1] M.S. Vitiello, G. Scalari, B. Williams. P. De Natale. Opt. Express, 23, 5167 (2015).

[2] E. Strupiechonski, D. Grassani, D. Fowler, F.H. Julien, S.P. Khanna, L. Li, E.H. Linfield, A.G. Davies, A.B. Krysa, Colombelli. Appl. Phys. Lett., 98, 101101 (2011).

[3] F. Stern. Phys. Rev. Lett., 18, 546 (1967).

[4] A.A. Dubinov, V.Ya. Aleshkin, V. Mitin, T. Otsuji, V. Ryzhii. J. Phys.: Condens. Matter, 23, 145302 (2011).

[5] Д.В. Ушаков, А.А. Афоненко, А.А. Дубинов, В.И. Гавриленко, О.Ю. Волков, Н.В. Щаврук, Д.С. Пономарев, Р.А. Хабибуллин. Квант. электрон., 49, 913 (2019).
[6] K. Hirakawa, H. Sakaki. Phys. Rev. B, 33, 8291 (1986).

Редактор А.Н. Смирнов

\section{Model of a terahertz quantum-cascade laser based on a two-dimensional plasmon}

\author{
A.A. Dubinov ${ }^{\mathbf{1 , 2}}$, V.Ya. Aleshkin ${ }^{\mathbf{1 , 2}}$ \\ ${ }^{1}$ Institute for Physics of Microstructures, \\ Russian Academy of Sciences, \\ 603950 Nizhny Novgorod, Russia \\ ${ }^{2}$ Lobachevsky State University of Nizhny Novgorod, \\ 603950 Nizhny Novgorod, Russia
}

Abstract In this work, we calculated the characteristics of a two-dimensional plasmon amplified by an active medium based on a terahertz quantum-cascade structure. It is shown that for realistic parameters of $\mathrm{GaAs} / \mathrm{AlGaAs}$ structures with quantum wells (mobility $2 \cdot 10^{5} \mathrm{~cm}^{2} \cdot \mathrm{V}^{-1} \cdot \mathrm{s}^{-1}$ at an electron concentration of $5 \cdot 10^{11} \mathrm{~cm}^{-2}$ and at temperatures up to $77 \mathrm{~K}$ ), the gain of a twodimensional plasmon can reach $1500 \mathrm{~cm}^{-1}$ for frequency $2.3 \mathrm{THz}$. In addition, due to the strong localization of the plasmon electric field near the quantum well, only a few cascades of the active medium are required for amplification. 